http://dx.doi.org/10.35381/racji.v5i3.1109

\title{
Principios de la Ley Orgánica del Sistema Nacional de Contratación Pública
}

Principles of the Organic Law of the National Government Procurement System

\author{
Alexandra Dolores Molina-Manzo \\ ub.alexandramolina@uniandes.edu.ec \\ Universidad Regional Autónoma de Los Andes, Babahoyo \\ Ecuador \\ https://orcid.org/0000-0001-5015-9739 \\ Giancarlos Rafael Guamán-Verdezoto \\ compraspangua@gmail.com \\ Universidad Regional Autónoma de Los Andes, Babahoyo \\ Ecuador
}

Recibido: 31 de octubre de 2020

Revisado: 10 de octubre de 2020

Aprobado: 05 de diciembre de 2020

Publicado: 10 de diciembre de 2020 


\title{
RESUMEN
}

La Contratación Publica en el Ecuador sufrió una drástica metamorfosis en el ámbito jurídico, tecnológico y social a partir del año 2008.El objetivo de esta investigación fue realizar un análisis sobre la Ley Orgánica del Sistema Nacional de Contratación Pública y su repercusión en los hechos de corrupción desde un enfoque cuantitativo con una metodología descriptiva con diseño no experimental, la cual se apoyó en el análisis documental -bibliográfico. Se ha empleado la técnica documental que nos ha permitido la recopilación de información para enunciar las teorías que sustentan el estudio de los principios de la contratación pública, así como su aplicación y cumplimiento. Lo cual se logró estructurar mediante el método analítico-sintético. Se concluye que el cumplimiento y aplicación de los principios de la contratación pública que se encuentran claramente establecidos en la Ley, deben ser de estricto cumplimiento por parte de los funcionarios involucrados en este ámbito y deben hacerse cumplir.

Descriptores: Administración pública; corrupción; aplicación de la ley. (Palabras tomadas del Tesauro UNESCO).

\begin{abstract}
Public Procurement in Ecuador underwent a drastic metamorphosis in the legal, technological and social fields as of 2008. The objective of this research was to carry out an analysis on the Organic Law of the National Public Procurement System and its repercussion on the events of corruption from a quantitative approach with a descriptive methodology with a non-experimental design, which was supported by the documentary-bibliographic analysis. The documentary technique has been used that has allowed us to collect information to state the theories that support the study of the principles of public procurement, as well as their application and compliance. Which was structured through the analytical-synthetic method. It is concluded that compliance and application of the principles of public procurement that are clearly established in the Law, must be strictly enforced by the officials involved in this area and must be enforced.
\end{abstract}

Descriptors: Public administration; corruption; application of the law. (Words taken from the UNESCO Thesaurus). 


\section{INTRODUCCIÓN}

La contratación pública es de trascendental importancia para el desarrollo y vigencia del Estado Ecuatoriano y para la consecución de los fines y objetivos que lo orientan. Sin embargo, la Ley de Contratación Pública, expedida el 16 de agosto de 1990 y codificada el 22 de febrero de 2001, no brindaba respuesta oportuna y eficiente a los requerimientos de obras, bienes y servicios de las entidades contratantes con parámetros de calidad y costo, ni fue una herramienta para optimizar los recursos públicos. En consecuencia, han sido frecuentes los casos de corrupción, paralización de obras, dotación de bienes y servicios de mala calidad y en definitiva desatención de las necesidades y requerimientos de la población, en particular de los sectores menos favorecidos.

A lo anterior hay que añadir que la Ley de Contratación Pública tenía un reducido ámbito de aplicación, debido a la proliferación de reglas que imperaban en el mismo ámbito, lo cual en la práctica hacía que solo se aplique en los ministerios y en determinados organismos seccionales. Sin embargo, las grandes contrataciones normalmente estuvieron exentas de su ámbito, tanto por la creación de leyes que determinaron regímenes especiales de contratación, como también porque la contratación de diversas instituciones y empresas, cuyo capital accionario es propiedad del Estado, se ha normado por regímenes distintos, autónomos, en el que ha primado la discrecionalidad y abuso en la toma de decisiones. Además, se vivía una gran inseguridad jurídica por cuanto los procesos de contratación de montos menores a los determinados para el concurso público de ofertas se sometían a la reglamentación especial de cada ente contratante.

La nueva Ley Orgánica del Sistema Nacional de Contratación Pública(LOSNCP), amplía su ámbito de acción y ahora recoge a todas las instituciones que según el artículo 225 de la Constitución de la República del Ecuador forman parte del sector público, y a todas aquellas entidades y empresas mercantiles que, aunque sean de derecho privado tiene en su patrimonio capitales públicos. Además, amplía su objeto 
de aplicación porque abarca a todas las contrataciones referentes a la adquisición de bienes, ejecución de obras, y prestación de servicios incluyendo los de consultoría. Sin embargo, la misma ley en el artículo 2 establece un régimen especial, en cuyo caso le corresponde al Presidente de la República emitir el correspondiente reglamento que regirá a los procedimientos precontractuales de dicho régimen.

Los principios establecidos en la Ley Orgánica del Sistema Nacional de Contratación Pública, así como los principios de todos los instrumentos legales son de estricto cumplimiento por parte de los integrantes de aquellos grupos, instituciones, regiones y demás que formen parte de dicho efecto jurídico, también son objeto de un debate al momento de su efectivo cumplimiento, ya que al igual que los demás principios establecidos en los diferentes instrumentos legales su cumplimiento efectivo es de vital importancia para el desarrollo de los entes que formen parte de su ámbito de aplicación.

El Servicio Nacional de Contratación Pública(SERCOP) como ente Rector de la Contratación Pública en el Ecuador, ha venido elaborando un marco jurídico y técnico con la finalidad de complementar las acciones de control; para los procesos de contratación y para lograr el cumplimiento de los principios establecidos en la Ley Orgánica del Sistema Nacional de Contratación Pública, lo cual de alguna manera ha ayudado a las instituciones determinadas en el Art. 1 de la ley anteriormente citada como una guía de uso y aplicación en el ejercicio de sus funciones y atribuciones.

Actualmente los procesos de contratación pública, han sido duramente señalados por las supuestas irregularidades que se han venido dando en las contrataciones realizadas por las instituciones públicas del país, durante la pandemia generada por el COVID-19 o Coronavirus, los más evidentes escándalos son los suscitados en los hospitales públicos del país, lo cual se evidencio y salió a la luz con trabajos investigativos de diferentes medios de comunicación, así como también han sido y son hasta la presente fecha noticia de todos los días, y sin lugar a dudas en las redes sociales este tipo de noticia han inundado este nuevo medio de comunicación. En este sentido Scheller \& Silva-Maestre, (2017), manifiestan: 
En un escenario donde se manejan grandes cantidades de dinero provenientes del erario, prima la discrecionalidad y la subjetividad de los individuos investidos por un cargo público o político fácilmente corruptible4, en el que además es fácil el acceso a actos fraudulentos; allí, existe una alta probabilidad de impunidad, por ello, resulta ser el escenario perfecto para cometer actos de corrupción. (p.8)

Es así, y a partir de lo anterior, que es necesario realizar un análisis técnico, legal, científico y de observación sin pasionismo, ni conveniente para ningún interés político ni menos aún en pretensión de protagonismo de ninguna manera. Pero un claro ejemplo se dio en la provincia de Manabí, cuando en el año 2016 tuvo que soportar un terremoto de magnitudes apocalípticas, quedando destruida su infraestructura vial y hospitalaria, al efecto se destinaron 124 millones de dólares para su reconstrucción, sin embargo, muy poco se ha avanzado y el dinero ya se ha gastado y la población manabita de Pedernales sufre el déficit hospitalario y por ende la falta de atención ante las emergencias de salud.( Zapata-Benavides, Arrias-Añez \& Aradia-Zambrano, 2020)

La Ley Orgánica del Sistema Nacional de Contratación Pública se expidió en el Registro Oficial No. 395 de 4 de agosto de 2008, en cuyo "Considerando" se resume el espíritu de la Ley en pro de dinamizar la Contratación Pública, mediante la innovación tecnológica que fomente la agilidad, eficiencia y trasparencia de los procesos de contratación; incentivo a la producción nacional; planificación y correcto uso de recursos públicos.

La Ley previó la necesidad de crear el Sistema Nacional de Contratación Pública(SNCP), cuya definición la encontramos en el artículo 7 de la citada norma: "el conjunto de principios, normas, procedimientos, mecanismos y relaciones organizadas orientadas al planteamiento, programación, presupuestos, control, administración y ejecución de las contrataciones realizadas por las Entidades Contratantes."

Con la expedición de la Ley Orgánica del Sistema Nacional de Contratación Pública, se derogó de manera expresa las siguientes normativas jurídicas: 
Tabla 1. Relación de normas derogadas.

\begin{tabular}{|c|l|}
\hline Norma jurídica & \multicolumn{1}{c|}{ Derogatoria } \\
\hline Ley de Contratación Pública & $\begin{array}{l}\text { Codificación Registro Oficial 272 de } \\
\text { 22 de febrero del 2001. }\end{array}$ \\
\hline Ley de Consultoría & $\begin{array}{l}\text { Publicada en el Registro Oficial } 455 \\
\text { de } 5 \text { de noviembre del 2004. }\end{array}$ \\
\hline $\begin{array}{c}\text { Codificación de la Ley Orgánica de la } \\
\text { Procuraduría General del Estado }\end{array}$ & $\begin{array}{l}\text { Letra f) del Art. 3 y letra b) del Art. 14 } \\
\text { publicada en el Registro Oficial 312 de } \\
13 \text { de abril de 2004. }\end{array}$ \\
\hline $\begin{array}{c}\text { Ley Orgánica de Administración } \\
\text { Financiera y Control. }\end{array}$ & Artículos 18 y 60 \\
\hline $\begin{array}{c}\text { Ley Orgánica de la Contraloría } \\
\text { General del Estado. }\end{array}$ & Numerales 16 y 35 del Art. 31 \\
\hline
\end{tabular}

Elaboración: Autores (2020).

La Ley Orgánica del Sistema Nacional de Contratación Pública, señala en el artículo 4, los principios que se deberán observar en materia de contratación pública:

Art. 4.- Principios. - Para la aplicación de esta Ley y de los contratos que de ella se deriven, se observarán los principios de legalidad, trato justo, igualdad, calidad, vigencia tecnológica, oportunidad, concurrencia, transparencia, publicidad; y, participación nacional.

Sin embargo, se han manifestado prácticas de corrupción vulnerando las leyes de República, ocasionando pérdidas al Estado y beneficios para la colectividad, al respecto Castañeda (2016:106) indica que el estudio de la corrupción requiere del trabajo multidisciplinar, pues la decisión de un agente de incurrir en actos de corrupción no solamente depende de la ganancia monetaria que pueda obtener, sino además del modo como la sociedad lo juzgue y la probabilidad de ser descubierto. En otros términos, la corrupción toma lugar bajo determinado marco institucional, de modo que el fenómeno no se extiende de manera homogénea entre países. Por ello es de suma importancia la participación de los diferentes actores de la administración pública y descentralizada para combatir esto hechos. 
El objetivo de esta investigación fue realizar un análisis sobre la Ley Orgánica del Sistema Nacional de Contratación Pública y su repercusión en los hechos de corrupción.

\section{METODOLOGÍA}

La investigación se desplegó desde un enfoque cuantitativo con una metodología descriptiva con diseño no experimental, la cual se apoyó en el análisis documental bibliográfico. Se realizó una exploración bibliográfica de modo metódico de la información existente a través de una minuciosa indagación, eligiendo y evaluando apropiadamente diferentes fuentes tales como: legislación ecuatoriana, articulo científicos y textos. Se ha empleado la técnica documental que nos ha permitido la recopilación de información para enunciar las teorías que sustentan el estudio de los principios de la contratación pública, así como su aplicación y cumplimiento. Lo cual se logró estructurar mediante el método analítico-sintético.

\section{RESULTADOS}

Se muestra un conjunto de resultados documentales:

Tabla 2.

\begin{tabular}{|c|c|}
\hline Base legal & Contenido e interpretación \\
\hline Artículo 204 de la Constitución & $\begin{array}{l}\text { El pueblo es el mandante y primer } \\
\text { fiscalizador del poder público, en } \\
\text { ejercicio de su derecho a la participación }\end{array}$ \\
\hline $\begin{array}{l}\text { Artículo } 212 \text { de Constitución } \\
\text { numeral } 2\end{array}$ & $\begin{array}{l}\text { Serán funciones de la Contraloría } \\
\text { General del Estado. } \\
\text { Determinar responsabilidades } \\
\text { administrativas y civiles culposas e } \\
\text { indicios de responsabilidad penal, } \\
\text { relacionadas con los aspectos sujetos a } \\
\text { su control, sin perjuicio de las funciones } \\
\text { que en esta materia sean propias de la } \\
\text { Fiscalía General del Estado. }\end{array}$ \\
\hline $\begin{array}{l}\text { Artículo } 16 \text { de Código Orgánico Integral } \\
\text { Penal }\end{array}$ & $\begin{array}{l}\text { Ámbito temporal de aplicación. - Los } \\
\text { sujetos del proceso penal y las o los } \\
\text { juzgadores observarán }\end{array}$ \\
\hline
\end{tabular}




\begin{tabular}{|c|c|}
\hline & $\begin{array}{l}\text { las siguientes reglas: } \\
\text { 1. Toda infracción será juzgada y } \\
\text { sancionada con arreglo } \\
\text { a las leyes vigentes al momento de su } \\
\text { comisión. } \\
\text { 2. Se aplicará la ley penal posterior más } \\
\text { benigna sin } \\
\text { necesidad de petición, de preferencia } \\
\text { sobre la ley penal } \\
\text { vigente al tiempo de ser cometida la } \\
\text { infracción o } \\
\text { dictarse sentencia. } \\
\text { 3. El ejercicio de la acción y las penas } \\
\text { prescribirán de } \\
\text { conformidad con este Código. } \\
\text { 4. Las infracciones de agresión a un } \\
\text { Estado, genocidio, } \\
\text { lesa humanidad, crímenes de guerra, } \\
\text { desaparición } \\
\text { forzada de personas, peculado, } \\
\text { cohecho, concusión, } \\
\text { enriquecimiento ilícito y las acciones } \\
\text { legales por daños } \\
\text { ambientales son imprescriptibles tanto } \\
\text { en la acción } \\
\text { como en la pena. }\end{array}$ \\
\hline Artículo 278 de COIP & $\begin{array}{l}\text { Artículo 278.- Peculado. - Las o los } \\
\text { servidores públicos y las personas que } \\
\text { actúen en virtud de una potestad estatal } \\
\text { en alguna de las instituciones del } \\
\text { Estado, determinadas en la Constitución } \\
\text { de la República, en beneficio propio o de } \\
\text { terceros; abusen, se apropien, distraigan } \\
\text { o dispongan arbitrariamente de bienes } \\
\text { muebles o inmuebles, dineros públicos o } \\
\text { privados, efectos que los representen, } \\
\text { piezas, títulos o documentos que estén } \\
\text { en su poder en virtud o razón de su } \\
\text { cargo, serán sancionados con pena } \\
\text { privativa de libertad de diez a trece años. }\end{array}$ \\
\hline Artículo 285 de COIP & $\begin{array}{l}\text { Tráfico de influencias. Las o los } \\
\text { servidores públicos, y las personas que } \\
\text { actúen en virtud de una potestad estatal } \\
\text { en alguna de las instituciones del }\end{array}$ \\
\hline
\end{tabular}




\begin{tabular}{|c|c|}
\hline & $\begin{array}{l}\text { Estado, enumeradas en la Constitución } \\
\text { de la República, prevaliéndose de las } \\
\text { facultades de su cargo o de cualquier } \\
\text { otra situación derivada de su relación } \\
\text { personal o jerárquica, ejerza influencia } \\
\text { en otra u otro servidor para obtener un } \\
\text { acto o resolución favorable a sus } \\
\text { intereses o de terceros, serán } \\
\text { sancionados con pena privativa de } \\
\text { libertad de tres a cinco años. }\end{array}$ \\
\hline $\begin{array}{l}\text { Artículo } 4 \text { de la Ley Orgánica del sistema } \\
\text { Nacional de Contratación Pública }\end{array}$ & $\begin{array}{l}\text { Para la aplicación de esta Ley y de los } \\
\text { contratos que de ella se deriven, se } \\
\text { observarán los principios de legalidad, } \\
\text { trato justo, igualdad, calidad, vigencia } \\
\text { tecnológica, oportunidad, concurrencia, } \\
\text { transparencia, publicidad; y, y, } \\
\text { participación nacional. }\end{array}$ \\
\hline
\end{tabular}

Elaboración: Autores (2020).

\section{Resultados del estudio realizado por la Secretaria Técnica de Transparencia}

Del $100 \%$ de denuncias recibidas, aproximadamente un $70 \%$ corresponden a temas de contratación pública, por presuntas irregularidades en las fases precontractual, contractual o de ejecución; identificándose los siguientes campos vulnerables:

- Alto índice de discrecionalidad en el manejo de contrataciones bajo Régimen Especial.

- Direccionamiento en TDRs y especificaciones técnicas.

- Ínfima cuantía, como medio para eludir contrataciones a gran escala.

- Porcentaje de subastas inversas por negociación son superiores a los de puja.

- Consultorías por contratación directa superan en número a los otros tipos contratación: lista corta y concurso público.

- Los casos de subcontratación, cuando llegan a ser por el 100\% del servicio ofertado, también constituyen otro ejemplo de análisis.

El fraude en la contratación pública, tiene varias presentaciones y pocas formas de identificarlo, orientadas a la corrupción que se caracterizan por sus modelos y estructuras atacando sectores vulnerables. 


\section{DISCUSIÓN}

La información obtenida al tratarse de normas legales vigentes e inherentes a los principios de la contratación pública en general, han sido de valiosa importancia ya que de las mismas se ha podido establecer un análisis crítico y objetivo.

Los principios de la contratación pública se encuentran claramente establecidos en la Ley Orgánica del sistema Nacional de Contratación Pública, su cumplimiento y aplicación debe ser de estricto cumplimiento por los funcionarios inmiscuidos en este ámbito y deben hacerse cumplir por las máximas autoridades de las entidades contratantes, así como por los entes de control.

Sin embargo, se existen grandes retos para evitar los auges de corrupción producto de las malas acciones en materia de contracion publica. Al respecto Fuerte-Carrera, Arrias-Añéz, Pupo-Kairuz (2020), manifiestan:

En los últimos años, el Ecuador ha estado sujeto a diversas políticas públicas ineficaces que han generado altos índices de desempleo, inseguridad y pobreza en detrimento del bienestar común. Los actos de corrupción generados como consecuencia de las irregularidades cometidas en los procesos de selección, contratación y ejecución de obras por parte del Estado desde el año 2000 han provocado entre otras cosas, instrucciones fiscales, detenciones, juicios, obras inconclusas, incautaciones, nuevos procesos licitatorios y de adjudicación directa, así como el cierre de entes públicos. (p.785).

Es necesario recalcar que no se puede generalizar, pero si se debe hacer un análisis profundo del personal que trabaja en este ámbito de la administración pública, pues al improvisar servidores $o$ al permitir que funcionarios manejen este tema de contratación pública en las entidades sin los debidos conocimientos y /o trabajen bajo presión de sus superiores siempre se estarán cometiendo infracciones de manera consiente e inconsciente. 


\section{CONCLUSIONES}

Los principios estipulados en el Art. 4 de la Ley Orgánica del sistema Nacional de Contratación Pública son claros, son precisos, los cuales cumplidos a cabalidad por las entidades contratantes lograrían conducir a la administración pública en un ejemplo de progreso. Se cumplen de manera parcial, muchas veces por falta de conocimiento de las personas al frente de una entidad contratante y otras porque se ha querido incumplirla para usar el sistema normativo a favor de intereses que no son netamente los institucionales.

El Sistema Nacional de contratación Pública es una herramienta informática, que al igual que cualquier sistema de esta índole es de fácil manipulación, ya sea legal o ilegalmente y queda comprobado con las diferentes veces que ha sido violada la seguridad del mismo, con la finalidad de beneficiar a uno o varios proveedores del estado.

Las máximas autoridades, representantes de un pueblo y todos quienes ejercen responsabilidad o están al frente de una entidad contratante, deben tener pleno conocimiento de la contratación pública y sus normas legales y sobre todo de los principios que rigen la misma, caso contrario solamente se dejaran conducir por personas que quizás no los puedan guiar de la manera correcta.

El cumplimiento y aplicación de los principios de la contratación pública que se encuentran claramente establecidos en la Ley, deben ser de estricto cumplimiento por parte de los funcionarios inmiscuidos en este ámbito y deben hacerse cumplir por las máximas autoridades de las entidades contratantes, así como por los entes de control.

\section{FINANCIAMIENTO}

No monetario.

\section{AGRADECIMIENTO}

A la Universidad Regional Autónoma de los Andes; por motivar el desarrollo de la Investigación. 


\section{REFERENCIAS CONSULTADAS}

Asamblea Nacional de la República del Ecuador. (2014). Código Orgánico Integral Penal. [Comprehensive Organic Criminal Code]. Recuperado de https://n9.cl/g6sc

Asamblea Nacional Constituyente de la República del Ecuador, (2008). Constitución de la República del Ecuador. Montecristi. Registro Oficial 449 de 20-oct-2008. Recuperado de https://n9.cl/sia

Castañeda-Rodríguez, V. (2016) Una investigación sobre la corrupción pública y sus determinantes. [An Inquiry on Public Corruption and its Determinants]. Revista Mexicana de Ciencias Políticas y Sociales.61(227),103-136. https://doi.org/10.1016/S0185-1918(16)30023-X

Congreso Nacional. (12 de junio de 2002). Ley Orgánica de la Contraloría General del Estado. Registro Oficial 595. Quito, Ecuador

Fuerte-Carrera, C., Arrias-Añéz, J. \& Pupo-Kairuz, A. (2020) Análisis sobre el peculado y la delincuencia organizada en los procesos de contratación pública emergente. [Analysis of peculate and organized crime in emerging public procurement processes]. Iustitia Socialis. 5(2), 783-797. http://dx.doi.org/10.35381/racji.v5i2.1065

Gobierno de la República del Ecuador (2008). Ley Orgánica del Sistema Nacional de Contratación Pública. [National Government Procurement System Organic Law] (registro Oficial Suplemento 395 del 04 de agosto de 2008). Recuperado en: www.lexis.com.ec

Gobierno de la República del Ecuador. Servicio Nacional de Contratación pública. https://portal.compraspublicas.gob.ec/sercop/\#

Secretaria Nacional de la Administración Pública. Investigación y Judicialización del Fraude y la Corrupción en la Contratación Pública. [Investigation and Judicialization of Fraud and Corruption in Government Procurement]. Recuperado de: https://n9.cl/ki20z

Scheller, A. \& Silva-Maestre, S. (2017) La corrupción en la contratación pública: operatividad, tipificación, percepción, costos y beneficios. [Corruption in government procurement: operability, typification, perception, costs and benefits] Revista VIA IURIS, 23,1-36 Recuperado de: http://www.redalyc.org/articulo.oa?id=273957284004 
Iustitia Socialis. Revista Arbitrada de Ciencias Jurídicas.

Año V. Vol. V. №3. Edición Especial. 2020-III:

Universidad Regional Autónoma de los Andes

Hecho el depósito de Ley: FA2016000064

ISSN: 2542-3371

FUNDACIÓN KOINONIA (F.K). Santa Ana de Coro, Venezuela

Alexandra Dolores Molina-Manzo; Giancarlos Rafael Guamán-Verdezoto

Zapata-Benavides, A., Arrias-Añez, J., \& Aradia-Zambrano, J. (2020). El delito del peculado y su impacto socioeconómico en el Ecuador. IUSTITIA SOCIALIS, 5(2), 748-758. doi:http://dx.doi.org/10.35381/racji.v5i2.1057

2020 por los autores. Este artículo es de acceso abierto y distribuido según los términos y condiciones de la licencia Creative Commons Atribución-NoComercial-Compartirlgual 4.0 Internacional (CC BY-NC-SA 4.0) (https://creativecommons.org/licenses/by-nc-sa/4.0/). 